\title{
Importancia de las estrategias didácticas y metodológicas en las dificultades de comprensión lectora, el lenguaje y comunicación
}

\section{Importance of didactic and methodological strategies in reading} comprehension, language and communication difficulties

Luis Oswaldo Jiménez Alarcón. ${ }^{1}$, Mónica Narciza López Pazmiño. ${ }^{2}$, Julio César Freire Pazmiño. ${ }^{3} \&$ Julio Rafael Cabrera López. ${ }^{4}$

\begin{abstract}
.
In the educational system different techniques, activities, actions, processes that benefit in the different areas of knowledge have been proposed; however, the application of strategies that reduce reading comprehension difficulties is scarce, which has increased deficiencies in decoding, vocabulary poverty, prior knowledge limitations, factors that influence memory problems. It is highlighted that since 2010, education in Ecuador is projected in the development of skills, abilities and skills of students, with support in critical pedagogy, with a constructive approach in cognitive ways, being the first actor of education His learning; considering that the instruction was based on the use of methodologies based on memorization, tasks and repetitive

\footnotetext{
${ }^{1}$ Escuela de Formación de Soldados del Ejército (Esforse). Ambato, Ecuador. luisojimenez1 @espe.edu.ec

${ }^{2}$ Universidad Tecnológica Indoamérica, Facultad de Ciencias Humanas, de la Educación y Desarrollo Social, Carrera de Educación Básica e Inicial. Ambato, Ecuador. monicalopez@uti.edu.ec

${ }^{3}$ Universidad Tecnológica Indoamérica, Facultad de Ciencias Humanas, de la Educación y Desarrollo Social, Carrera de Educación Básica e Inicial. Ambato, Ecuador. juliofreire@uti.edu.ec

${ }^{4}$ Universidad Tecnológica Indoamérica, Facultad de Ciencias Humanas, de la Educación y Desarrollo Social, Carrera de Educación Básica e Inicial. Ambato, Ecuador. juliocabrera@uti.edu.ec
} 
exercises, added to the precarious use of guidelines in the field of teaching and methodology that strengthen the acquisition of reading habits. From this vision, the study called: Teaching and methodological strategies that reduce difficulties in reading comprehension, language and communication, aims to determine its incidence in low self-esteem, disinterest in the study and insecurity. In the methodology the qualitative and quantitative approach is used, with the collection of relevant and field bibliographic information, the survey technique was applied, with the structured questionnaire instrument, which allows interacting with reality; in addition the independent and dependent variable is determined; Subsequently, the analysis and interpretation of data obtained that benefit in the verification of the hypothesis, through the statistical test of Chi2, with a significance of 0.05 and 6 degrees of freedom; deducing that the methodology applied by the teacher does not allow an active participation of students, damaging academic performance, communication, dialogue and interaction, deteriorating the teaching-learning process; In addition, the degree of reading comprehension is meager, there are conflicts in deduction, argumentation, intellectual, emotional and social development, factors that limit educational quality.

Keywords: strategies, methodological, communication, reading comprehension, language, interaction.

\section{Resumen.}

En el sistema educativo se han propuesto diferentes técnicas, actividades, acciones, procesos que benefician en las diversas áreas del conocimiento; sin embargo es escasa la aplicación de estrategias que disminuyan las dificultades de comprensión lectora lo que ha incrementado deficiencias en la decodificación, pobreza en el vocabulario, limitaciones conocimientos previos, factores que influyen en los problemas de memoria. Se resalta que desde el año 2010, la educación en el Ecuador se proyecta en el desarrollo de capacidades, habilidades y destrezas de los educandos, con sustento en la pedagogía crítica, con un enfoque constructivo en vías cognitivas, siendo el educando el primer actor de su aprendizaje; considerando que la instrucción se fundamentaba en la utilización de metodologías basadas en la memorización, tareas y ejercicios repetitivos, sumado al precario empleo de orientaciones en el campo de la didáctica y metodología que fortalezcan la adquisición de hábitos lectores. Desde esta visión, el estudio denominado: Estrategias didácticas y metodológicas que disminuyen las dificultades en la comprensión lectora, el lenguaje y comunicación, tiene por objeto determinar su incidencia en la baja autoestima, desinterés en el estudio e inseguridad. En la metodología se utiliza el enfoque de carácter cualitativo y cuantitativo, efectuándose la recolección de información bibliográfica pertinente y de campo, se aplicó la técnica de la encuesta, con el instrumento cuestionario 
estructurado, que permite interactuar con la realidad; además se determina la variable independiente y dependiente; posteriormente se efectúa el análisis e interpretación de datos obtenidos que beneficio en la comprobación de la hipótesis, a través de la prueba estadística del Chi2, con una significación de 0.05 y 6 grados de libertad; deduciéndose que la metodología aplicada por el docente no permiten una participación activa de los estudiantes, perjudicando en el rendimiento académico, la comunicación, el diálogo e interacción, deteriorando el proceso de enseñanza aprendizaje; además el grado de comprensión lectora es exiguo, existiendo conflictos en la deducción, argumentación, el desarrollo intelectual, emocional y social, factores que limitan la calidad educativa.

Palabras claves: estrategias, metodológica, comunicación, comprensión lectora, lenguaje.

\section{Introducción.}

Todas las personas al ser entes sujetos para aprender se encuentran involucrados en el sistema educativo y formativo, mediante el proceso enseñanza-aprendizaje aporta en la adquisición de conocimientos, habilidades y actitudes para enfrentar y resolver problemas concretos en diferentes tipos de contextos; es decir en la enseñanza estudiante fortalece el pensamiento lógico, crítico y creativo, de esta manera se cumple con los principios de equidad e inclusión propuestos en la Ley de Educación.

En el Ecuador, la Constitución en su artículo 26, manifiesta que "La educación es un derecho de las personas a lo largo de su vida y deber ineludible e inexcusable del Estado..." y mediante el Acuerdo Ministerial No. 0482-12, se expidió por primera vez en el país Estándares Educativos. Con el ACUERDO N. MINEDUC-ME-2016-00020-A., se ajusta los estándares de aprendizaje para las áreas de Matemática, Lengua y Literatura, Ciencias Naturales y Ciencias Sociales. Estos estándares de aprendizaje tienen como propósito orientar, apoyar y monitorear la acción de los actores del sistema educativo hacia su mejora continua. Adicionalmente, los acuerdos ofrecen insumos para la toma de decisiones de políticas públicas que mejoran de la calidad del sistema educativo. Como se evidencia los diferentes componentes del quehacer educativo, con el empleo de la normativa se enfocan al mejoramiento continuo.

"La concepción de enseñar y aprender en el país ha sido en los últimos años una constante incertidumbre, pues ha traído consigo una serie cambios que no han beneficiado a la enseñanza ecuatoriana. La revolución educativa de la cual tanto se habla, no se ha podido evidenciar en los salones de clase” (Benavides \& Henríquez, 2018). 
Según El (Instituto Nacional de Estadísticas y Censos, 2012) a nivel nacional en referencia a la adquisición de hábitos de lectura se obtiene los porcentajes: el 27\% de habitantes no tiene hábito de leer, el $57 \%$ demuestra desinterés, el $32 \%$ no lee por falta de tiempo, el 3\% presenta problemas de concentración.

En cuanto a los hábitos de lectura por ciudad la población de Guayaquil y Ambato leen en un $77 \%$, Cuenca en el 68\%; con una frecuencia del $31 \%$ el sector poblacional mantiene su lectura en los periódicos en su tiempo libre, el 28\% en libros. Según lo expuesto, (Barreiro, 2017) afirma que los organismos internacionales como la UNESCO fortalecen la idea que la inclusión educativa se sustenta en los derechos humanos, en el acceso y participación a una educación de calidad; en este sentido los docentes mediante capacitaciones buscan mejorar la labor pedagógica.

El (Ministerio de Desarrollo Social, 2016) menciona en los componentes prioritarios las bases curriculares en el área de lenguaje y comunicación, propone ejes de formación que deben ser alcanzados por los educandos en cada nivel de escolarización de la enseñanza básica sobresale que el ser humano construye y comprende el mundo que le rodea al entrar en diálogo consigo mismo y con personas del contexto. El (Ministerio de Educación, 2012) a través de los avances tecnológicos actualiza a los docentes en el manejo de recursos electrónicos, aportando en la lectura y escritura en combinación con las estrategias didácticas y metodológicas beneficia en la comprensión lectora, la decodificación, el vocabulario y entendimiento; desde esta percepción se compromete en fortalecer el rendimiento y desempeño académico. Además, las adaptaciones y el uso de herramientas de apoyo en el entorno escolar y extraescolar permiten el alcance de niveles aceptables en el proceso de lectoescritura.

Un aspecto trascendental en el desarrollo de estrategias didácticas y metodológicas de apoyo al aprendizaje, comprensión lectora, el vocabulario, la comunicación influye en la capacidad intelectual, la expresión, atención, actitudes de iniciativa, conducta, sociabilidad; de igual manera contribuye en los procesos de memoria a corto y largo plazo en correlación con el análisis el pensamiento creativo y procesos del lenguaje expresivo.

Según (El Telégrafo, 2018), la población lectora del país en un 77\% mantiene una baja relación en la lectura. Los adolescentes de 12 a 16 años no asisten a bibliotecas y no utilizan el fondo bibliográfico lo que disminuye la utilización de libros perjudicando en el bagaje cultural en un mundo globalizado por esta razón se considera necesario que durante el ciclo de educación básica los estudiantes a través de estrategias didácticas y metodológicas desarrollen saberes cognitivos y no cognitivos que encaminen a la autonomía y participación en la sociedad. 
Para (Ausubel, 1983) el pensamiento es progresivo e independiente por tal razón los programas de estudio apoyan al desarrollo de habilidades de lenguaje, escrito, hablado y razonamiento. (Arceo \& Hernández, 1998) menciona que en el área de lenguaje y comunicación la utilización de estrategias didácticas y metodológicas aportan en la investigación y evaluación (Aldana, Vereda, Hidalgo-Alvarez, \& de Vicente, 2016) afirma que en el aprendizaje es importante alcanzar una actitud reflexiva y analítica que favorece en las aptitudes que benefician en la participación de forma responsable y activa en una sociedad libre y democrática al generar en los estudiantes una disposición positiva hacia el saber, (Avanzini, 2016) es así como despierta la curiosidad e interés en el entorno que le rodea; de esta forma se incentiva en la búsqueda de información, se ejercita la creatividad, la iniciativa y confianza en sí mismo para enfrentar situaciones resolver problemas y tomar decisiones.

(Barreiro, 2017), las bases curriculares en el área de lengua y comunicación definen los desempeños mínimos que deben ser alcanzados por los educandos. Los objetivos integran el desarrollo de habilidades relevantes para alcanzar el crecimiento integral; de esta manera adquieren las herramientas necesarias que favorecen en la toma de decisiones. (Bossis, Marins, Kuzhir, Volkova, \& Zubarev, 2015), en éste sentido el currículo es flexible y permite la adaptación a realidades educativas desde diferentes enfoques educativos familiar social (Song, King, Yoon , Cho, \& Jeong, 2014) aproximaciones curriculares, didácticas y metodológicas que orientan en la adquisición de conocimientos, que conlleva al desarrollo armónico de habilidades cognitivas, el vocabulario, la pronunciación, la comprensión, el discernimiento y argumentación.

\section{Metodología.}

Toda investigación requiere el reconocimiento y definición de la metodología empleada en la investigación, a través del cual se aproxime al objeto de estudio. La indagación actual su accionar se dirige a recabar información de la fuente primaria y someterla a un estudio estadístico que determina la esencia del fenómeno causa efecto con respaldo en la teoría existente, se utilizó el enfoque cualitativo y cuantitativo.

En el enfoque cualitativo se "utiliza la recolección y análisis de particularidades que mejoran las preguntas de investigación o revelan nuevas interrogantes en el proceso de interpretación." (Hernández Sampieri, Fernández Collado, \& Baptista Lucio, 2014, pág. 7) Por consiguiente, se pretende es describir las cualidades de un fenómeno mediante el análisis y comprensión de fenómenos. En la orientación cuantitativa, "emplea la recolección de datos que comprueba la hipótesis con base en la medición numérica y el análisis estadístico, con el fin establecer pautas de actuación y probar teorías." (2014, pág. 4); plantear un problema específico y su subsecuente hipótesis incluye las variables que serán sujetas a comprobación numérica, se podrá deducir la relación causa-efecto, obteniendo en el estudio la objetividad, como única forma de alcanzar el conocimiento, intentando buscar la certeza del mismo. 
Se emplea la modalidad de investigación documental, se realizó apoyándose en fuentes de información de naturaleza secundaria en concordancia con el problema, acción que facilitó la comprensión y su posterior análisis e interpretación; la segunda es la indagación de campo, se obtuvo la información directamente de la realidad obteniendo un conocimiento profundo del inconveniente motivo del estudio. En el nivel exploratorio se familiarizó e identificó los hechos que permitieron conocer las condiciones necesarias y suficientes para efectuar la observación; las variables involucradas fueron las estrategias didácticas - metodológicas.

Nivel Descriptivo, Se describe cómo es y cuándo se manifiesta el problema, en sus características, situaciones o contextos en los que se producen; se recabó datos de las variables; ¿qué se va a medir? (estrategias didácticas - metodológicas y la comprensión lectora) y sobre quienes (estudiantes, padres de familia y profesores).

Investigación Correlacional, busca establecer la relación o grado de asociación existente entre las variables, sometiéndose a examinar la hipótesis formulada, utilizando el método del Chi-Cuadrado, conforme lo manifiesta (Hernández Sampieri, Fernández Collado, \& Baptista Lucio, 2014, pág. 318).

Nivel explicativo, el interés fue estudiar las causas que producen una anomalía, en otras palabras, se explica ¿por qué ocurre un fenómeno? y ¿en qué condiciones se manifiesta? o ¿por qué se relacionan las variables?, siendo necesario explicar ¿por qué se tiene un bajo nivel de comprensión lectora? y ¿en qué condiciones se manifiesta?, además el conocer ¿por qué se relacionan las estrategias didácticas - metodológicas con las deficiencias en la comprensión lectora, lenguaje, la comunicación, decodificación, el vocabulario y expresión en los estudiantes.

Población y muestra a fin de acercarnos a la realidad es necesario convertir los diferentes conceptos o variables en elementos operativos y manejables, procediéndose a su determinación:

"La población, o en términos precisos se obtiene:

Población objetivo, es un conjunto finito o infinito de elementos con características comunes siendo extensivas las conclusiones de la investigación, delimitada por el problema y por los objetivos del estudio" (Arias, 2012, pág. 81); el área poblacional fue definida por 128 personas; constituidas por docentes, estudiantes y padres de familia de educación general básica media de la Escuela de educación Básica Manuela Espejo. Se considera el criterio de Sabino, 1992; "una muestra, en un sentido amplio, no es más que eso, una parte del todo llamado universo y que sirve para representarlo" (1992, pág. 91); por lo expuesto la muestra es probabilista estratificada (aleatoria), se "divide a la población en diferentes grupos, con el 
único fin es obtener la representatividad a los diversos grupos en cuestión" (Gómez Bastar, 2012, pág. 34); describiéndose:

Tabla 1. Población objeto de estudio.

\begin{tabular}{lcc}
\hline Población objeto de estudio & Frecuencia & Porcentaje \\
\hline Docentes & 8 & $16 \%$ \\
Estudiantes & 22 & $44 \%$ \\
Padres de familia & 20 & $40 \%$ \\
\hline \multicolumn{1}{c}{ Totales } & $\mathbf{5 0}$ & $\mathbf{1 0 0 \%}$ \\
\hline
\end{tabular}

Fuente: Elaboración propia.

De esta manera se emplea el muestreo probabilístico estratificado, se establece con un nivel de confianza del 95\%, un error estadístico máximo del 5\% una significación del 0,05\%; considerando que la muestra probabilística es estratificada y aleatoria porque contribuye al cumplimento de los objetivos planteados en la indagación, obteniendo información teórica y numérica que realza el conocimiento adquirido en base a la realidad de la institución.

En la identificación de variables: independiente estrategias didácticas y metodológicas, a través de su conceptualización como categorías: proceso enseñanza aprendizaje (participación, construcción de conocimientos); capacidades (autonomía, práctica de valores); y resolver problemas (responsabilidad social). Dependiente, comprensión lectora, a través de su conceptualización: comprensión (toma de conciencia, dominio del conocimiento); construcción (habilidades sociales, vocabulario); y pensamiento (crítico).

Al operacionalizar las variables, se determinó la encuesta como técnica válida en la recolocación de información de campo, empleándose el instrumento el cuestionario estructurado, con preguntas cerradas, dirigido a docentes, estudiantes y padres de familia de la Escuela de Educación Básica Manuela Espejo, del cantón Ambato; que facilitó el registro de hechos y aspectos coherentes con las estrategias didácticas - metodológicas y deficiencia en la comprensión lectora.

De acuerdo con lo manifestado por Avila, 2006 "la encuesta se utiliza para estudiar poblaciones mediante muestras representativas a fin de explicar las variables de estudio y su frecuencia" (pág. 55), recabando datos de carácter cuantitativo, a través de preguntas escritas, redactadas en un instrumento denominado cuestionario, que facilitó la codificación, el observación y la interpretación de los datos. 
Las interrogantes estructuradas fueron diseñadas para la recolección de datos específicos, pertinente el conocimiento del fenómeno a investigar, sus aspectos y elementos de mayor relevancia; precisándose en las preguntas que deben aplicarse a los docentes, estudiantes y padres de familia de la Escuela de Educación Básica Manuela Espejo, acciones que conllevan a la comprobación de la hipótesis planteada; en el diseño del cuestionario se toma el criterio de Corral, 2010 "las interrogaciones estructuradas presentan un grupo de alternativas de respuesta, ya preestablecidas" (pág. 6), la opción múltiple (ofrece una serie de respuestas donde el participante selecciona una o más alternativas) o dicotómicas (con dos alternativas de respuesta), herramienta aplicada en la obtención de información clara, oportuna e interesante para dar solución al problema planteado.

La validez y confiabilidad de los instrumentos aplicados se realizó con la técnica juicio de expertos; siendo analizados por especialistas en investigación y en el área de estudio, emitieron criterios sobre su contenido, pertinencia, ambigüedad y redacción. Al cumplirse este procedimiento, las observaciones y sugerencias, se produjo el rediseño final del instrumento.

Tabla 2. Items junto con las alternativas de elección; instrumento (cuestionario aplicado a la muestra objeto de estudio)

\begin{tabular}{cl}
\hline No. & Preguntas \\
\hline $\mathbf{1}$ & $\begin{array}{l}\text { Participa activamente, demuestra sus conocimientos, experiencias y facultades en la } \\
\text { búsqueda de soluciones a problemas del diario vivir }\end{array}$ \\
$\mathbf{2}$ & $\begin{array}{l}\text { Aportar en la construcción conocimientos mediante juegos, lecturas, que favorecen la } \\
\text { comprensión, el descubrimiento y la libre expresión }\end{array}$ \\
$\mathbf{3}$ & Mediante estrategias fortalece el vocabulario, la memoria y decodificación \\
$\mathbf{4}$ & Fortalece el interés por la lectura la autoestima desde un aspecto interactivo \\
$\mathbf{5}$ & Exterioriza un escaso control y dirección del proceso lector \\
$\mathbf{7}$ & $\begin{array}{l}\text { Material bibliográfico que aporte en la adquisición de conocimientos, en la toma de } \\
\text { conciencia, la sensibilización cultural y social }\end{array}$ \\
$\mathbf{8}$ & Estimula habilidades sociales, hábitos de trabajo y la creatividad \\
$\mathbf{9}$ & $\begin{array}{l}\text { Desarrolla la observación, atención } \\
\text { la ortografía }\end{array}$ \\
\end{tabular}

Fuente: Elaboración propia. 
Al efectuar la verificación de la hipótesis se emplea la prueba del Chi-cuadrado, observándose si dos parámetros o variables están o no relacionadas; es decir, son independientes o no son independientes la una de la otra.

Las variables serán las estrategias didácticas-metodológicas con la deficiencia en comprensión lectora, en la determinación se seleccionaron seis interrogantes de la encuesta aplicada a la muestra objeto de estudio.

Para la comprobación de la hipótesis se empleó el modelo lógico y el modelo estadístico: Hipótesis nula $\left(\mathrm{H}_{0}\right)$ : La utilización de estrategias didácticas y metodológicas no disminuye las dificultades en comprensión lectora, el lenguaje y comunicación en los estudiantes de la Escuela de Educación Básica Manuela Espejo.

Hipótesis alternativa (H1): La utilización de estrategias didácticas y metodológicas si disminuye las dificultades en comprensión lectora, el lenguaje y comunicación.

En el modelo estadístico se empleó la fórmula de Chi- cuadrado, cuya fórmula menciona la sumatoria de frecuencias observadas menos las frecuencias esperadas divididas para las frecuencias esperadas, con un nivel de significación de 0.05 , el $95 \%$ de confiabilidad y 10 grados de libertad.

Se acepta la hipótesis nula (Ho) si el valor de Chi-cuadrado teórico es mayor o igual al valor de Chi-cuadrado calculado $\left(\mathrm{X}_{\mathrm{t}}^{2} \geq \mathrm{XC}^{2}\right)$.

Se rechaza la hipótesis nula (Ho) y se aceptará la hipótesis alternativa (H1) si el valor del Chi-cuadrado calculado es mayor al Chi-cuadrado teórico $\left(\mathrm{XC}^{2}>\mathrm{X}_{\mathrm{t}}{ }^{2}\right)$. Por lo expuesto, el valor de Chi-cuadrado teórico $\left(\mathrm{X}_{\mathrm{t}}{ }^{2}\right)$ con 10 grado de libertad y un nivel de significación de 0,05 de 18.31, cantidad obtenida en la tabla de puntos porcentuales de la distribución Chi-cuadrado.

Para determinar la relación de las variables objeto de estudio se seleccionaron dos preguntas de la encuesta aplicada a docentes, estudiantes y padres de familia de la Escuela de Educación Básica Manuela Espejo, como se puede apreciar en la tabla de frecuencias observadas, existen dos preguntas para cada extracto de muestra analizada; lo que facilitó la realización del proceso de verificación y comprobación de la hipótesis.

Tabla 3. Chi Cuadrado Calculado X 2

\begin{tabular}{crrrc}
\hline FO & FE & $($ Fo - Fe $)$ & $(\mathbf{O}-\mathbf{E}) \mathbf{2}$ & $(\mathbf{F O}-\mathbf{F E}) \mathbf{2} / \mathbf{F E}$ \\
\hline 1 & 1,52 & $-0,52$ & 0,3 & 0,18 \\
1 & 1,52 & $-0,52$ & 0,3 & 0,18
\end{tabular}




\begin{tabular}{|c|c|c|c|c|}
\hline 7 & 4,18 & 2,82 & 8,0 & 1,90 \\
\hline 2 & 4,18 & $-2,18$ & 4,8 & 1,14 \\
\hline 5 & 3,8 & 1,2 & 1,4 & 0,38 \\
\hline 3 & 3,8 & $-0,8$ & 0,6 & 0,17 \\
\hline 7 & 4,96 & 2,04 & 4,2 & 0,84 \\
\hline 4 & 4,96 & $-0,96$ & 0,9 & 0,19 \\
\hline 15 & 13,64 & 1,36 & 1,8 & 0,14 \\
\hline 14 & 13,64 & 0,36 & 0,1 & 0,01 \\
\hline 13 & 12,4 & 0,6 & 0,4 & 0,03 \\
\hline 9 & 12,4 & $-3,4$ & 11,6 & 0,93 \\
\hline 0 & 1,52 & $-1,52$ & 2,3 & 1,52 \\
\hline 3 & 1,52 & 1,48 & 2,2 & 1,44 \\
\hline 0 & 4,18 & $-4,18$ & 17,5 & 4,18 \\
\hline 6 & 4,18 & 1,82 & 3,3 & 0,79 \\
\hline 2 & 3,8 & $-1,8$ & 3,2 & 0,85 \\
\hline 8 & 3,8 & 4,2 & 17,6 & 4,64 \\
\hline 100 & 100 & 0 & 80,5 & 19,5 \\
\hline
\end{tabular}

Fuente: Elaboración propia.

Se observa, con diez grados de libertad un nivel de significación de $\alpha=0.05$; el Chi-cuadrado calculado es mayor que el Chi-cuadrado teórico, esto otras palabras se rechaza la hipótesis nula (Ho) y se acepta la alternativa (H1) que dice: las estrategias didácticas y metodológicas si disminuyen las dificultades en comprensión lectora, el lenguaje y comunicación en los estudiantes de Educación General Básica en consecuencia existe relación entre la variable independiente y dependiente. 
Tabla 4. Interpretación Chi Cuadrado Calculado X 2

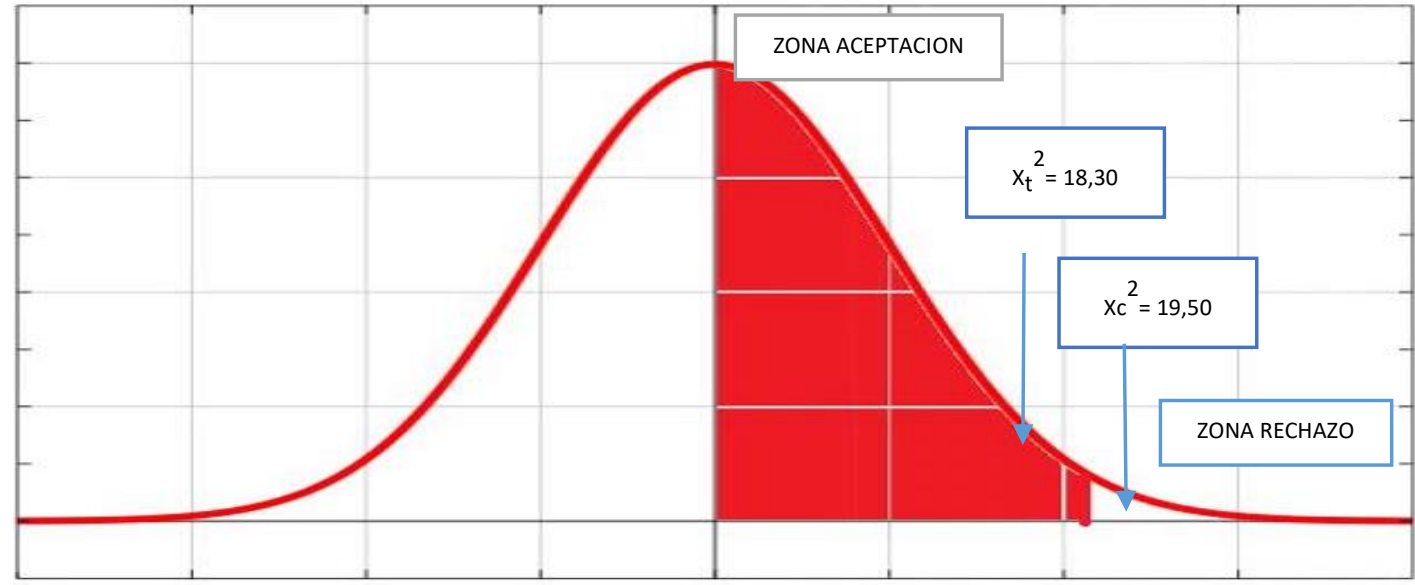

Fuente: Elaboración propia.

Se deduce que a veces el docente aplica estrategias fortalece el vocabulario, la memoria y decodificación; es decir no despierta en el educando el interés por la lectura la autoestima desde un aspecto interactivo.

Se identificó que el docente a veces utiliza estrategias metodológicas lo que limita la participación activa, la interacción, el trabajo en equipo, perjudicando en el rendimiento escolar, en la comunicación, el diálogo, lo que deteriora el proceso de enseñanza-aprendizaje, el cambio de ideas, experiencias, valores nuevos conocimientos, la capacidad de acceder, comprender y reflexionar sobre cualquier tipo de información, desfavoreciendo en la creatividad, el entendimiento y la toma decisiones.

Se establece que el nivel de comprensión lectora es insuficiente, ya que el educando demuestra una actitud pasiva cuando lee, llevando a una actividad rutinaria, carente de esfuerzo hacia la búsqueda y construcción del significado, lo que perjudica en la práctica de valores, sobresaliendo la utilización de libros para impartir el conocimiento, estableciendo conflictos en el entendimiento, la comunicación, el análisis y la argumentación, la capacidad de razonamiento, reduciendo la autoestima, autorrealización, identificación de logros y limitaciones.

\section{Discusión.}

Utilizar estrategias didácticas y metodológicas para lograr la participación activa, el trabajo en equipo, mejorando el rendimiento escolar, la comunicación, el diálogo y la interacción a través del fortalecimiento del proceso enseñanza - aprendizaje, el cambio de ideas, experiencias, valores nuevos conocimientos, la capacidad de acceder, comprender y 
reflexionar sobre cualquier tipo de información, favoreciendo en la creatividad, el entendimiento y la toma decisiones.

Fortalecer el nivel de comprensión lectora, apoyando al educando en la educación en la creatividad, en el esfuerzo hacia la búsqueda y construcción del significado, incrementando la práctica de valores, el conocimiento, la comprensión, el entendimiento, la comunicación, el análisis, la argumentación, la capacidad de razonamiento, la autoestima, autorrealización para alcanzar la identificación de logros y limitaciones.

Promover el desarrollo intelectual, emocional, social, los hábitos de trabajo, las habilidades reflexivas, criticas, incrementando la adquisición del vocabulario, la ortografía, y las conexiones con la estructura cognitiva, impulsando al educando hacia el desarrollo intelectual, emocional, la concentración, la comunicación, el diálogo y el trabajo en equipo.

La comprensión lectora se caracteriza por ser un proceso interactivo entre el lector y el texto (Beltrán, 2009), se constituye en un tema de interés en el sistema educativo y la sociedad de la información que cada vez con el adelanto de la ciencia es más exigente al considerarse como un procedimiento constructivo (Barona, 2010); las capacidades comprensivas trascienden desde la atención, la percepción, la memoria, el reconocimiento, la identificación hasta lograr niveles pensamiento crítico y creativo (Cadena, 2015) apoya en la extracción de información, la inferencia e interpretación de aspectos que permiten establecer relaciones lógicas (frases y párrafos); esta forma los individuos fortalecen la capacidad individual en el análisis de textos escritos que beneficien el desarrollo de conocimientos.

El ser humano se encuentra inmerso en las redes de la comunicación personal interpersonal, intrapersonal e intragrupal (Calderón, 2018), beneficia en el diálogo, la socialización, la solución de problemas mediante el razonamiento y reflexión; (Bello, Naranjo, Cano, \& Sánchez, 2017); el lenguaje incluye la conversación, los actos de habla, el discurso que benefician la lingüísticas y producción de significados, se presentan en la comunicación oral o verbal (2017); desde el bagaje cognoscitivo y vivencial la competencia comunicativa interviene en la conciencia de formación el proceso de aprendizaje, elementos que permiten el intercambio de ideas y representación de la realidad.

En la metalingüística la comprensión lectora, el lenguaje y comunicación contribuyen en la función expresiva donde el ser humano emite opiniones y vivencias como manifestación subjetiva de la realidad siendo un proceso interactivo e interpersonal que desde las perspectivas semántica, neurológica, psicológica, cultural y social influye en las actividades mentales y emocionales. 


\section{Conclusiones:}

- Utilizar estrategias metodológicas y didácticas disminuyan los problemas de comprensión lectora, los escasos conocimientos previos, las deficiencias en codificación, la pobreza de vocabulario, las dificultades de memoria ,el desinterés por realizar las tareas; además, emplear actividades que reduzcan la confusión, el desconocimiento y escaso dominio en la adquisición de saberes apoyando al educando en la educación en la creatividad, en el esfuerzo hacia la búsqueda y construcción del significado, incrementando la práctica de valores, el conocimiento, el entendimiento, la comunicación, el análisis, la argumentación, la capacidad de razonamiento, la autoestima, autorrealización para alcanzar la identificación de logros y limitaciones.

- Utilizar estrategias que favorezcan en la metacognición: mapas, gráficos, organizadores; contestación de preguntas, resumen; decorar el aula con dibujos, realizar una cartelera efectuar inferencias, determinar las partes importantes de un texto, visualizar imágenes emplear pictogramas, elementos que influyen en el cambio de ideas, experiencias, valores nuevos conocimientos, la capacidad de acceder, comprender y reflexionar sobre cualquier tipo de información, favoreciendo en el proceso lector, en la creatividad, el entendimiento y la toma decisiones.

- Aplicar actividades que promuevan la lectura de forma divertida, leer en voz alta algunas partes del libro describir lo que le agradó de la historia decorar el aula con dibujos realizar una cartelera efectuar inferencias determinar las partes importantes de un texto visualizar imágenes emplear pictogramas

- Establecer como reto alcanzar cambios significativos que mejoren el aprendizaje colaborativo, el dominio del código lingüístico, la competencia dialógica, responsabilidad, el desarrollo intelectual, emocional, social, los hábitos de trabajo, las habilidades reflexivas, criticas, incrementando la adquisición del vocabulario, la ortografía, y las conexiones con la estructura cognitiva, impulsando al educando el diálogo y el trabajo en equipo.

\section{Referencias bibliográficas.}

Aldana, S., Vereda, F., Hidalgo-Álvarez, R., \& de Vicente, J. (2016). Facile synthesis of magnetic agarose microfibers by directed selfassembly. Polymer, 93, 61-64.

Arceo, D. B., \& Hernández, R. G. (1998). Estrategias docentes para un aprendizaje significativo una interpretación constructivista. Mc Graw Hill.

Arias, F. (2012). El Proyecto de Investigación. Introducción a la metodología científica. Caracas: Episteme.

Ausubel, D. (1983). Teoría del aprendizaje significativo. (https://s3.amazonaws.com/academia.edu.documents/38902537/Aprendizaje_signifi 
cativo.pdf?AWSAccessKeyId=AKIAIWOWYYGZ2Y53UL3A\&Expires $=1553926$ 948\&Signature=yldSZtQnoQ2p1mJih7yQ0EdDC2U\%3D\&response-contentdisposition=inline\%3B\%20filename\%3DTEORIA_DEL_APRENDIZJ, Ed.) Trillas, $1(1), 10$.

Avanzini, G. (2016). Problemas de Pedagogía. El Fracaso escolar. (https://www.amazon.com/El-Fracaso-Escolar-Guy-Avanzini/dp/B072K9FWMN, Ed., \& L. Medrano, Trad.) Barakaldo, España: Herder, S.A.

Avila, H. (2006). Introducción a la Metodología de la Investigación. Chihuahua: Edición electrónica.

Barona, D. (2010). La teoría de la Inteligencia Emocional enfatiza la participación de las emociones para solucionar conflictos que se presentan en los distintos contextos de la vida diaria. Barcelona: Paidós.

Barreiro, P. L. (2017). Perspectivas metodológicas en la enseñanza y en la investigación en educación matemática. Universidad Nacional de General Sarmiento, 8.

Bello, M., Naranjo, N., Cano, J., \& Sánchez, D. (5 de Junio de 2017). Perfil Cognitivo y Psicolingüístico y su Relación con la Lectoescritura en un Preescolar con Síndrome de Down. (https://www.aidep.org/sites/default/files/2018-04/RIDEP47-Art9.pdf, Ed.) Revista Iberoamericana de Diagnóstico y Evaluación - e Avaliação Psicológica. RIDEP , 2(47), 16.

Bello, Z., Rionda, D., \& Rodríguez, M. (2010). Inteligencia y pedagogía para el desarrollo emocional de los educandos. Barcelona, España: Paidós.

Beltrán, J. (2009). Estrategias de aprendizaje significativo. Madrid, España: Eudema.

Benavides, P., \& Henríquez, S. (2018). Incidencia de las estrategias metodológicas en el proceso de lecto escritura de los estudiantes de educación básica elemental de la unidad educativa Réplica Aguirre Abad. Santiago de Chile, Chile: Universidad de Concepción Campus Los Ángeles Escuela de Educación. Obtenido de http://repositorio.udec.cl/bitstream/handle/11594/2508/Benavides\%20$\% 20 \mathrm{Henr} \% \mathrm{C} 3 \%$ ADquez.pdf?sequence=1\&isAllowed=y

Bossis, G., Marins, J., Kuzhir, P., Volkova, O., \& Zubarev, A. (2015). Functionalized microfibers for field-responsive materials and biological applications. Journal of Intelligent Material Systems and Structures, 1-9.

Cadena, M. (2015). La comprensión lectora y su influencia en el aprendizaje significativo de los estudiantes del Séptimo año de Educación General Básica Paralelo "B" de la Escuela de Educación Fe y Alegría, Ambato provincia de Tungurahua. 
(http://repositorio.uta.edu.ec/bitstream/123456789/12713/1/FCHE-EBP-1424.pdf, Ed.) Ambato, Ecuador: Universidad Técnica de Ambato.

Caisatoa, S. d. (2012). Técnicas activas de aprendizaje y su incidencia en rendimiento académico del Área de Ciencias Naturales (Entorno Natural) de los estudiantes de segundo y tercer año de educación básica de la Escuela Fiscal Mixta Benjamin Carrión, parroquia Amaguaña. Ambato, Ecuador: Universidad Técnica de Ambato.

Calderón, D. (2018). Didáctica de lenguaje y comunicación: campo de investigación y formación de profesores. Praxis \& Saber, 9(21), 151-178.

Celi, C., \& Gonzales, G. (2018). Estrategias metodológicas centradas en el aprendizaje significativo. Latacunga, Ecuador: http://repositorio.utc.edu.ec/bitstream/27000/4444/1/PI-000598.pdf.

Corral, Y. (2010). Diseño de Cuestionarios para Recolección de Datos. REVISTA CIENCIAS DE LA EDUCACIÓN, 17.

Daza, O. (2011). Tareas escolares para el rendimiento académico de los infantes. Madrid, España: Barcelona.

El Telégrafo. (30 de Abril de 2018). Los datos de lectura en el país no son claros. Cultura, pág. 1. Obtenido de https://www.eltelegrafo.com.ec/noticias/cultura/10/datos-inechabitos-lectura-ecuador

Estrada Guerrero, R., Lemus Torres, D., Mendoza Anaya, D., \& Rodriguez Lugo, V. (2010). Hidrogeles poliméricos potencialmente aplicables en Agricultura. Revista Iberoamericana de Polímeros, 12(2), 76-87.

García-Cerda, L., Rodríguez-Fernández, O., Betancourt-Galindo, R., Saldívar-Guerrero, R., \& Torres-Torres, M. (2003). Síntesis y propiedades de ferrofluidos de magnetita. Superficies y Vacío., 16(1), 28-31.

Gómez Bastar, S. (2012). Metodología de la Investigación. Estado de México: RED TERCER MILENIO S.C.

Hernández Sampieri, R., Fernández Collado, C., \& Baptista Lucio, M. (2014). Metodología de la Invesigación. Mexico D. F.: McGRAW-HILL.

Ilg, P. (2013). Stimuli-responsive hydrogels cross-linked by magnetic nanoparticles. Soft Matter, 9, 3465-3468.

Instituto Nacional de Estadísticas y Censos. (2012). Hábitos de lectuar en Ecuador. Quito, Ecuador: Instituto Nacional de Estadísticas y Censos. Obtenido de 
http://www.celibro.org.ec/web/img/cms/ESTUDIO\%20HABITOS\%20DE\%20LEC TURA\%20INEC.pdf

Lewitus, D., Branch, J., Smith, K., Callegari, G., Kohn, J., \& Neimark, A. (2011). Biohybrid carbon nanotube/agarose fibers for neural tissue engineering. Advanced Functional Materials, 21, 2624-2632.

Lin, Y.-S., Huang, K.-S., Yang, C.-H., Wang, C.-Y., Yang, Y.-S., Hsu, H.-C., . . Tsai, C.W. (2012). Microfluidic synthesis of microfibers for magnetic-responsive controlled drug release and cell culture. PLoS ONE, 7(3), 1-8.

Ministerio de Desarrollo Social. (2016). Educación, síntesis de resultados. Quito, Ecuador: Ministerio de Desarrollo Social, Subsecretaría de Evaluación Social 29 de diciembre de 2016. Obtenido de http://observatorio.ministeriodesarrollosocial.gob.cl/casenmultidimensional/casen/docs/CASEN_2015_Resultados_educacion.pdf

Ministerio de Educación. (2012). Lenguaje y Comunicación. Santiago de Chile, Chile: https://www.curriculumnacional.cl/614/articles-18871_programa.pdf.

Ruiz Estrada, G. (2004). Desarrollo de un Sistema de liberación de fármacos basado en nanopartículas magnéticas recubiertas con Polietilénglicol para el tratamiento de diferentes enfermedades. Madrid: Universidad Autónoma de Madrid. Departamento de Física Aplicada.

Sabino, C. (1992). EL PROCESO DE INVESTIGACION. Bogotá: Ed. Panamericana.

Song , J., King, S., Yoon , S., Cho, D., \& Jeong, Y. (2014). Enhanced spinnability of narbon nanotube fibers by surfactant addition. Fiberes and Polymers, 15(4), 762-766.

Tartaj, P., Morales, M., González-Carreño, T., Veintemillas-Verdaguer, S., \& Serna, C. (2005). Advances in magnetic nanoparticles for biotechnology applications. Journal of Magnetism and Magnetic Materials, 290, 28-34.

Wulff-Pérez , M., Martín-Rodriguez, A., Gálvez-Ruiz, M., \& de Vicente, J. ( 2013 ). The effect of polymer surfactant on the rheological properties of nanoemulsions. Colloid and Polymer Science, 291, 709-716.

Zamora Mora, V., Soares, P., Echeverria, C., Hernández, R., \& Mijangos, C. (2015). Composite chitosan/Agarose ferrogels for potential applications in magnetic hyperethermia. Gels., 1, 69-80.

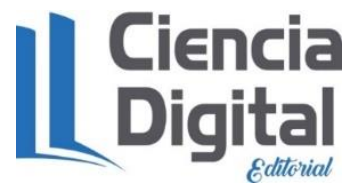


PARA CITAR EL ARTÍCULO INDEXADO.

Jiménez Alarcón, L. O., López Pazmiño, M. N., Freire Pazmiño, J. C., Cabrera López, J. R., \& Robles Portugal, G. K. (2020). Importancia de las estrategias didácticas y metodológicas en las dificultades de comprensión lectora, el lenguaje y comunicación. Explorador Digital, 4(3), 184-200. https://doi.org/10.33262/exploradordigital.v4i3.1322

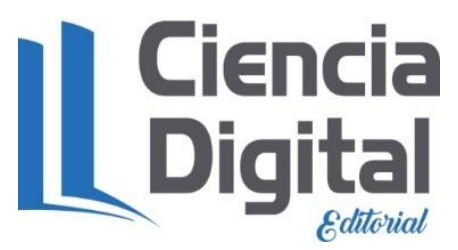

El artículo que se publica es de exclusiva responsabilidad de los autores y no necesariamente reflejan el pensamiento de la Revista Explorador Digital.

El artículo queda en propiedad de la revista y, por tanto, su publicación parcial y/o total en otro medio tiene que ser autorizado por el director de la Revista Explorador Digital.
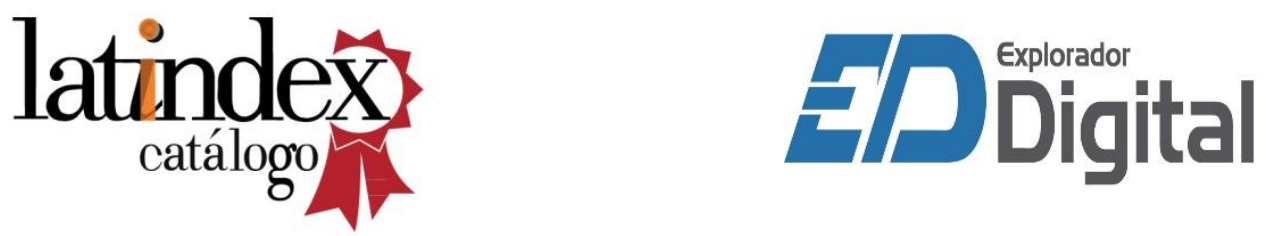